\title{
CUOTAS DE GÉNERO, LEYES ELECTORALES Y ELECCIÓN DE LEGISLADORAS EN AMÉRICA LATINA*
}

\author{
Gender Quotas, Electoral Laws, and the Election of Female \\ Legislators in Latin America
}

\section{MARK P. JONES}

Rice University

\section{SANTIAGO ALLES}

Rice University

\section{CAROLINA TCHINTIAN}

Rice University

\begin{abstract}
RESUMEN
El presente artículo utiliza datos a nivel de distrito de las elecciones de legisladores nacionales en 19 países de América Latina para evaluar el efecto de las cuotas de género, en conjunto con otras reglas electorales, sobre la elección de mujeres legisladoras. El trabajo actualiza y amplía un estudio previo utilizando datos correspondientes al último turno de renovación legislativa en los mismos 19 países. La implementación de cuotas de género adecuadamente diseñadas tiene un fuerte impacto positivo en la elección de mujeres: cuando cuotas bien diseñadas están en vigencia, el uso de listas cerradas da lugar a un mayor porcentaje de legisladoras electas (respecto a las listas abiertas), debido principalmente a la superioridad de los sistemas de listas cerradas cuando un partido logra dos asientos en un distrito.
\end{abstract}

Palabras clave: Reglas electorales, cuotas de género, legislaturas, representación de mujeres.

\begin{abstract}
This study employs district-level data from national legislative elections in 19 Latin American countries to evaluate the effect of gender quota legislation, in concert with other electoral rules, on the election of women legislators. It updates and extends a previous study on the same topic using data from more recent elections in the same 19 countries. Well designed quota legislation has a profound positive impact on the election of women. Where well designed quota legislation is in force, the use of closed lists on average results in a greater percentage of women legislators elected to office than does the use of open lists, primarily due to the marked superiority of the closed list systems when the number of seats a party wins in a district is two.
\end{abstract}

Key words: Electoral laws, gender quotas, legislatures, women's representation.

* Los autores agradecen a los expositores y asistentes del panel "Gender Quotas and Electoral Systems", en el encuentro de Midwest Political Science Association 2012, como así también a Tiffany Barnes, Allyson Benton, Akitaka Matsuo, William Reed, y los evaluadores anónimos por los comentarios, sugerencias y asistencia brindados en la elaboración del presente artículo. 


\section{CUOTAS DE GÉNERO, LEYES ELECTORALES Y ELECCIÓN DE LEGISLADORAS EN AMÉRICA LATINA}

La igualdad entre hombres y mujeres en la participación en la vida política ha ocupado un lugar relevante en la agenda de las Naciones Unidas durante más de treinta años. En esta dirección, la Convención sobre la Eliminación de Todas las Formas de Discriminación contra la Mujer (CEDAW, por su sigla en inglés), aprobada en 1979 y en vigor desde 1981, brinda un excelente ejemplo de la importancia que el sistema internacional reconoce a la igualdad global de las mujeres. La Cuarta Conferencia Mundial de las Naciones Unidas sobre la Mujer, celebrada en Beijing en 1995, puso de relieve la dramática subrepresentación de las mujeres en los órganos de decisión y abogó por la adopción de una serie de medidas para corregir esta falta de representación.

La subrepresentación de las mujeres, en particular en las legislaturas, es criticada por dos razones principales. Por un lado, la insuficiente representación descriptiva de las mujeres manifiesta una grave falla en el funcionamiento de una democracia (véase Bacchi, 2007; Dahlerup, 2006; Ríos Tobar, 2008). Es difícil, si no imposible, considerar a una democracia representativa funcionando a un nivel óptimo cuando más de la mitad de su población está insuficientemente representada en el Congreso. Por otro lado, numerosos estudios demuestran que existen diferencias significativas entre hombres y mujeres legisladores, tanto en su comportamiento legislativo como en sus preferencias políticas (véase, por ejemplo, Franceschet et al., 2012; Marx et al., 2007; Schwindt-Bayer, 2010). La evidencia indica que las legisladoras hacen una contribución importante y distinta a la representación en el Congreso (Norris, 2004).

En una dirección similar, Schwindt-Bayer (2007) ofrece una revisión excelente y concisa del impacto de las mujeres legisladoras en el proceso de políticas públicas en América Latina. Schwindt-Bayer señala que, además de servir como importantes e influyentes modelos de conducta para las mujeres (especialmente las jóvenes) en las sociedades latinoamericanas, las mujeres miembros del Congreso han tenido un impacto relevante en el proceso legislativo. Primero, las mujeres "han hecho importantes contribuciones en numerosas partes del proceso político, desde las preocupaciones y actitudes políticas que introducen en la agenda, pasando por las comisiones en las que participan impulsando legislación, hasta las políticas que patrocinan e impulsan" (Schwindt-Bayer, 2007: 4, nuestra traducción). En particular, Schwindt-Bayer identifica los avances en áreas tales como los derechos de las mujeres o legislación relacionada a infancia y familia, como ejemplos muy visibles de la repercusión sustantiva de la mujer en el proceso político en la región, con las mujeres legisladoras como responsables de avances significativos en las políticas durante los últimos años en áreas de vital importancia.

La adopción de políticas de acción afirmativa para aumentar el número de mujeres en cargos públicos fue una de las recomendaciones más importantes surgidas en la Conferencia de Beijing (Naciones Unidas, 1995). Esta recomendación, sin embargo, en ninguna parte del mundo ha sido implementada tan extensamente como en América Latina, donde catorce países han adoptado legislación exigiendo porcentajes mínimos de 
mujeres en las listas de candidatos de legisladores nacionales. ${ }^{1}$ No obstante, el porcentaje de mujeres legisladoras en muchos de estos países sigue siendo muy bajo a pesar de la adopción de estas medidas, con sólo un puñado de casos que pueden considerarse éxitos indiscutidos. La actual subrepresentación de las mujeres en las legislaturas de América Latina, tal como se discutirá más adelante, puede enfrentarse por medio de la adopción de una serie de reformas en la legislación electoral de esos países. En este terreno la evidencia es clara: si se desea aumentar en el corto y mediano plazo la proporción de mujeres en el Congreso, la introducción de leyes de cuotas bien concebidas representa la única opción viable para convertir este deseo en una realidad.

¿Qué factores condicionan la efectividad de la implementación de las cuotas de género? En un trabajo anterior sobre la relación entre cuotas, reglas electorales y la elección de mujeres en las legislaturas nacionales en América Latina, el primero de este tipo utilizando datos a nivel de distrito, Jones (2009) concluyó que la eficacia de las cuotas se ve afectada por las características del sistema electoral. En este artículo se reevalúan los resultados del trabajo anterior, analizando el ciclo más reciente de elecciones legislativas nacionales en la región. Utilizando nuevos datos, nuestro análisis en la mayoría de los casos confirma las conclusiones principales alcanzadas por Jones. En particular, los resultados aquí presentados ponen de relieve que cuotas de género bien concebidas tienen un impacto óptimo en la elección de mujeres cuando se utilizan junto con listas cerradas y en distritos donde los partidos políticos ganan más de un solo escaño. Al mismo tiempo, sin embargo, otros resultados difieren parcialmente de los obtenidos anteriormente, en especial en cuanto a la eficacia relativa de las cuotas de género frente a diferentes tipos de listas partidarias (cerradas vs abiertas).

El artículo continúa en el siguiente orden. En el próximo apartado se revisa la literatura sobre sistemas electorales y leyes de cuotas. Luego, se describe la población estudiada; y a continuación se detallan las variables (independientes y dependientes) utilizadas en el estudio. Por último, en las secciones finales se presenta el análisis empírico y luego se discuten sus implicancias teóricas.

\section{TIPO DE LISTAS, LEYES DE CUOTAS, MAGNITUD PARTIDARIA Y ELECCIÓN DE MUJERES}

La introducción de cuotas de género ha sido considerada como un fast-track (Dahlerup y Freidenvall 2005) hacia la igualdad entre géneros. En este terreno existe un extendido consenso en la literatura sobre los efectos positivos de las cuotas sobre las oportunidades electorales de las mujeres (Norris, 2004; Jones, 2004; Htun y Jones, 2002; Larserud y Taphorn, 2007; Matland, 2005; Tripp y Kang, 2008; Dahlerup, 1998; IDEA, 2003). No obstante, los mismos autores también destacan cómo otros elementos del diseño electoral pueden ser

1 La adopción de cuotas en América Latina ha sido un proceso dominado por la élite, con poca participación popular en la elaboración, debate e implementación de estas reformas. A su vez, tampoco existe una relación significativa entre el porcentaje de mujeres en la legislatura nacional antes de la primera adopción de las cuotas en Argentina en 1991 y la adopción posterior de cuotas de género en un país de América Latina. 
relevantes para potenciar (o atenuar) sus efectos: la eficacia de la legislación de cuotas es, tal como señalan Htun y Jones (2002), mayor cuando se combina con listas cerradas, mandatos de posición, distritos electorales medianos o grandes, y normas estrictas para asegurar su cumplimiento. La implementación de cuotas bajo ese diseño institucional tiene la ventaja de garantizar un piso mínimo de representación de las mujeres en todos los partidos y distritos.

El acceso de las mujeres a cargos legislativos en los sistemas de listas cerradas, una vez que la magnitud partidaria llega a dos o tres escaños, tiene un piso mínimo de entre el $20 \%$ y el $50 \%$, dependiendo de la magnitud de los partidos y las reglas electorales específicas de cada país. Por el contrario, el acceso de las mujeres en los sistemas de listas abiertas no tiene un piso asegurado, por lo cual su valor mínimo potencial es siempre $0 \%$. Sobre esta base, la mayor parte de la literatura (Htun y Jones, 2002; Krook, 2009; Larserud y Taphorn, 2007; Norris, 2004; Paxton et al., 2010; Schwindt-Bayer, 2010, entre otros) sostiene que, ceteris paribus, la combinación de cuotas con mandatos de posición y listas de candidatos cerradas debe dar lugar a un mayor número de mujeres legisladoras electas que cuando las cuotas son implementadas sin mandatos de ubicación en sistemas de listas abiertas. No obstante, la literatura no es unánime en este sentido: más recientemente, algunos trabajos (Matland, 2006; Schmidt, 2009) han puesto en duda la supuesta superioridad de la combinación de cuotas y listas cerradas.

En ausencia de cuotas de género, el efecto de la utilización de listas abiertas o cerradas sobre la elección de mujeres depende en gran medida de la actitud hacia la inclusión de mujeres entre las élites partidarias que deciden la composición de la listas (Larserud y Taphorn, 2007; Matland, 2006; Schmidt, 2009): si las élites partidarias son más progresistas que los votantes del partido, entonces las mujeres tienen más probabilidades de ser elegidas en una competencia con listas cerradas; mientras que, por el contrario, se verán más beneficiadas por las listas abiertas si las bases electorales del partido son más progresistas que sus élites. Los resultados de Thames y Williams (2010), sin embargo, sugieren que las listas cerradas son, ceteris paribus, en promedio más propicias para la elección de mujeres que las listas abiertas.

Por último, con independencia del tipo de la lista utilizada o la presencia/ausencia de leyes de cuotas, hay un consenso general de que cuanto mayor sea el número de escaños que un partido gana en un distrito electoral (es decir, la magnitud partidaria), mayor es la proporción de esos escaños que serán ocupados por mujeres (Larserud y Taphorn, 2007; Matland, 2005; Norris, 2004). Por esta razón (aunque no exclusivamente, véase Matland, 2005) los sistemas de representación proporcional son ampliamente reconocidos como aquellos más propicios para la elección de las mujeres por sobre las reglas de simple pluralidad en distritos uninominales o de los sistemas mayoritarios en dos vueltas (Larserud y Taphorn, 2007; Matland, 2005). Los sistemas de representación proporcional (con un moderado a alto número de legisladores electos por distrito) se asocian a magnitudes partidarias de moderadas a altas, mientras que en distritos uninominales, por definición, la magnitud partidaria será ' 1 ', porque ese es el número máximo de asientos que un partido puede ganar en el distrito. 
Si bien hay un extendido consenso acerca de la relación (positiva) entre magnitud partidaria y la elección mujeres, si esta relación es lineal, logarítmica, o sigue algún otro patrón es una pregunta para la cual la literatura existente ofrece aún pocas pistas. Más aún, es probable que esta relación varíe dependiendo del tipo de lista utilizado y de si las cuotas han sido implementadas o no por medio de legislación adecuadamente diseñada (Matland, 2006).

\section{DISEÑO DE INVESTIGACIÓN}

\section{Casos y tipos de cuotas}

El presente trabajo analiza, con datos al nivel de distrito, la última renovación legislativa en las democracias de América Latina. Dado que nuestro objetivo principal es observar la interacción entre cuotas de género y tipos de listas, antes que confirmar el hecho extensamente aceptado de que las mujeres tienen menos probabilidades de ser electas en distritos uninominales que en plurinominales (véase Larserud y Taphorn, 2007; Matland, 2005; Norris, 2004), la inclusión de las elecciones en distritos uninominales no es especialmente relevante. ${ }^{2}$ Como consecuencia de ello, las elecciones en distritos uninominales en los sistemas mixtos de Bolivia, México, Panamá, Puerto Rico y Venezuela han sido excluidos del análisis, al igual que las elecciones de senadores en Brasil (cuando sólo hay un escaño en juego) y en República Dominicana, como así también un pequeño número de casos de otros países donde la magnitud de los distritos fue uno. ${ }^{3}$

El diseño de investigación se ve condicionado tanto por la experiencia relativamente corta de las cuotas en la región, como así también por los límites en la disponibilidad de datos. Por esta razón, el número de observaciones disponibles por país es suficientemente restringido como para que cualquier ganancia en términos del tamaño de la $\mathrm{N}$ al reunir todas las elecciones de la región, sea contrarrestado por los problemas generados por la necesidad de controlar la naturaleza longitudinal de los datos. Por lo tanto, el presente análisis incluye un corte transversal de sólo el ciclo más reciente ( $\mathrm{al} 1^{\circ}$ de marzo de 2012) de renovación legislativa nacional. En total, el trabajo incluye elecciones de 29 cámaras legislativas en 19 países.

Una gran mayoría de los países de la región, tal como se observa en la Tabla 2, emplean reglas proporcionales en distritos plurinominales para elegir a los miembros de la Cámara de Diputados y el Senado. No obstante, algunos casos no se ajustan a esa regla general.

2 Bolivia brinda un contraste extremo de los efectos de leyes de cuotas eficaces en distritos plurinominales, respecto a sistemas con cuotas laxas o sin cuotas en distritos uninominales: aproximadamente la mitad de los diputados son elegidos en distritos uninominales (con cuotas laxas) y la otra mitad de distritos plurinominales con representación proporcional (cuotas de 50\% + mandatos de posición). En 2009, sólo el 9\% de los diputados electos en distritos uninominales fueron mujeres, frente al $41 \%$ en los distritos plurinominales.

3 El análisis de los determinantes de la elección de mujeres en todos los distritos donde un partido obtuvo un solo escaño (incluyendo distritos uninominales) demuestra que la exclusión de los distritos uninominales no afecta a las principales conclusiones alcanzadas aquí con respecto a la elección de las mujeres cuando magnitud partidaria es uno. Con la excepción de los distritos uninominales en México (en forma parcial) y Bolivia, las leyes de cuotas no se aplican a distritos uninominales en la región. 
Tabla 1: Elecciones legislativas incluidas, por tipo de Cámara, en 19 democracias

\begin{tabular}{lll}
\hline \multicolumn{1}{c}{ Unicameral } & \multicolumn{1}{c}{ Diputados } & \multicolumn{1}{c}{ Senado } \\
\hline Costa Rica 2010 & Argentina 2009-2011 & Argentina 2007-2009-2011 \\
Ecuador 2009 & Bolivia 2009 & Bolivia 2009 \\
El Salvador 2009 & Brasil 2010 & Brasil 2006*-2010 \\
Guatemala 2011 & Chile 2009 & Chile 2005*-2009 \\
Honduras 2009 & Colombia 2010 & Colombia 2010 \\
Nicaragua 2011 & República Dominicana 2010 & República Dominicana 2010 \\
Panamá 2009 & México 2009 & México 2006* \\
Perú 2011 & Paraguay 2008 & Paraguay 2008 \\
Venezuela 2010 & Puerto Rico 2008 & Puerto Rico 2008 \\
& Uruguay 2010 & Uruguay 2010 \\
\hline
\end{tabular}

Nota: Cuatro Cámaras legislativas tienen un sistema de renovación parcial: la Cámara de Diputados y el Senado en Argentina, el Senado brasileño, y el Senado en Chile. En esos casos se incluyeron múltiples elecciones a fin de abarcar el conjunto de los legisladores electos en la renovación. Todas las elecciones aquí incluidas son distintas de las incluidas en Jones (2009), con la excepción de aquellas indicadas con un asterisco en el texto: Senado de México, la mitad de las observaciones del Senado de Chile, y una tercera parte de las observaciones del Senado para Brasil. No obstante, dado que un tercio de los senadores brasileños electos en 2006 fueron elegidos de distritos uninominales, esas observaciones fueron excluidas del análisis principal del artículo.

Primero, todos los senadores en Argentina y las tres cuartas partes de los senadores en México son electos en distritos de tres escaños según una fórmula semiproporcional en la que el partido más votado obtiene dos asientos y el segundo, el restante. Segundo, las Cámaras de Diputados de Bolivia y México como así también las legislaturas unicamerales de Panamá y Venezuela utilizan diferentes variantes de representación proporcional mixta (MMPR, por la sigla en inglés); ${ }^{4}$ por lo cual, sólo se han incluido las elecciones de los distritos plurinominales bajo representación proporcional de este grupo. Por último, el Senado de Puerto Rico se elige por medio de un sistema de voto único no-transferible (SNTV, por la sigla en inglés) en distritos plurinominales; al tiempo que una pequeña proporción de los escaños en la Cámara de Diputados de Puerto Rico, una cuarta parte de la Asamblea de Venezuela, así como todo el Senado brasileño (en el turno de renovación cuando cada estado renueva dos senadores) son elegidos en distritos plurinominales por pluralidad.

Los congresos latinoamericanos presentan además variación en el tipo de listas de candidatos para la elección de sus miembros en los distritos plurinominales. Las listas cerradas se utilizan en la Argentina, Bolivia, Costa Rica, El Salvador, Guatemala, México, Nicaragua, Paraguay, Uruguay y los distritos proporcionales en Venezuela.

4 El sistema electoral en Panamá, en realidad, no es mixto en la medida que no hay dos niveles separados. No obstante, una proporción anormalmente grande de escaños bajo representación proporcional (37\%) son elegidos en distritos uninominales. 
Por el contrario, Brasil, Chile, República Dominicana, Ecuador, Honduras, Panamá, Perú, Puerto Rico, y los distritos de mayoría en Venezuela utilizan listas abiertas. Colombia permite a los partidos políticos determinar el formato de lista distrito a distrito, aunque en la práctica las listas abiertas dominan el panorama electoral: el 96\% de los legisladores electos en 2010 obtuvieron sus escaños con este tipo de listas. No obstante, entre los sistemas de listas abiertas existe alguna variación. Por un lado, Chile, Colombia, República Dominicana, los diputados en Brasil y los escaños electos por SNTV del Senado en Puerto Rico permiten a los votantes un voto de preferencia dentro de un mismo partido. ${ }^{5}$ Por otro, la elección de los senadores en Brasil, de los escaños electos por pluralidad en Venezuela y Puerto Rico, y de los miembros de la Asamblea en Panamá permiten a los votantes tantos votos de preferencia como escaños a cubrir dentro de un mismo partido. Perú permite a los votantes dos votos de preferencia (ya sea para uno o dos candidatos) dentro de un mismo partido, mientras que Ecuador y Honduras permiten a los votantes emitir tantos votos preferenciales como escaños haya por cubrir, sin estar limitados a hacerlo en un mismo partido (por ejemplo, panachage). ${ }^{6} \mathrm{~A}$ diferencia de los sistemas de listas abiertas en la mayor parte de Europa, en los sistemas latinoamericanos de listas abiertas no hay un listado ya ordenado por el partido político que sólo puede ser modificado por un número significativo de electores que utilizan la opción de voto preferencial.

De los diecinueve países, once emplean alguna forma de la legislación nacional de cuotas para al menos una de sus Cámaras legislativas: Argentina (Diputados, Senado), Bolivia (Diputados, Senado), Brasil (Diputados), Costa Rica, República Dominicana (Diputados), Ecuador, Honduras, México (Diputados, Senado), Panamá, Paraguay (Diputados, Senado), y Perú. ${ }^{7}$ Estos sistemas se pueden dividir en tres categorías generales: (a) casos en los que un porcentaje mínimo de mujeres debe aparecer en la lista e incluyen mandatos de posición, (b) casos en los que se exige un porcentaje mínimo de mujeres en las listas, pero no hay mandatos de posición en vigor (donde se incluyen los sistemas de listas abiertas, que son incompatibles con los mandatos de posición), y (c) casos en los que, en la práctica, no se exige ningún porcentaje mínimo de mujeres en las listas, no hay mandatos de posición o, más en general, la legislación de cuotas tiene fallas en su diseño, tal como se detalla más abajo. Los porcentajes mínimos exigidos por la legislación en los dos primeros grupos oscilan entre el $30 \%$ y el $50 \%{ }^{8}$

5 Si bien El Salvador no empleó listas abiertas en la elección incluida en este análisis (2009), en la actualidad utiliza este tipo de lista.

6 En los sistemas de listas abiertas de América Latina, los votantes emiten su voto de preferencia(s) ya sea marcando una casilla que aparece junto al nombre de un candidato (y, en varios países, la imagen) o escribiendo el número del candidato en la boleta electoral.

7 Además de estos países, tanto en Colombia y Uruguay han aprobado una legislación de cuotas de género que estará en vigencia para las próximas elecciones, que se celebrarán en 2014 y 2015, respectivamente. Hasta la reforma constitucional de 1999, Venezuela tuvo leyes de cuotas (aunque laxas).

8 Dado el rango limitado del porcentaje mínimo exigido por las leyes de cuotas ( $30 \%$ a $50 \%$ ) como a cuestiones relacionadas con la introducción de efectos fijos (fixed effects), no es posible incluir esta variable en el análisis principal. 


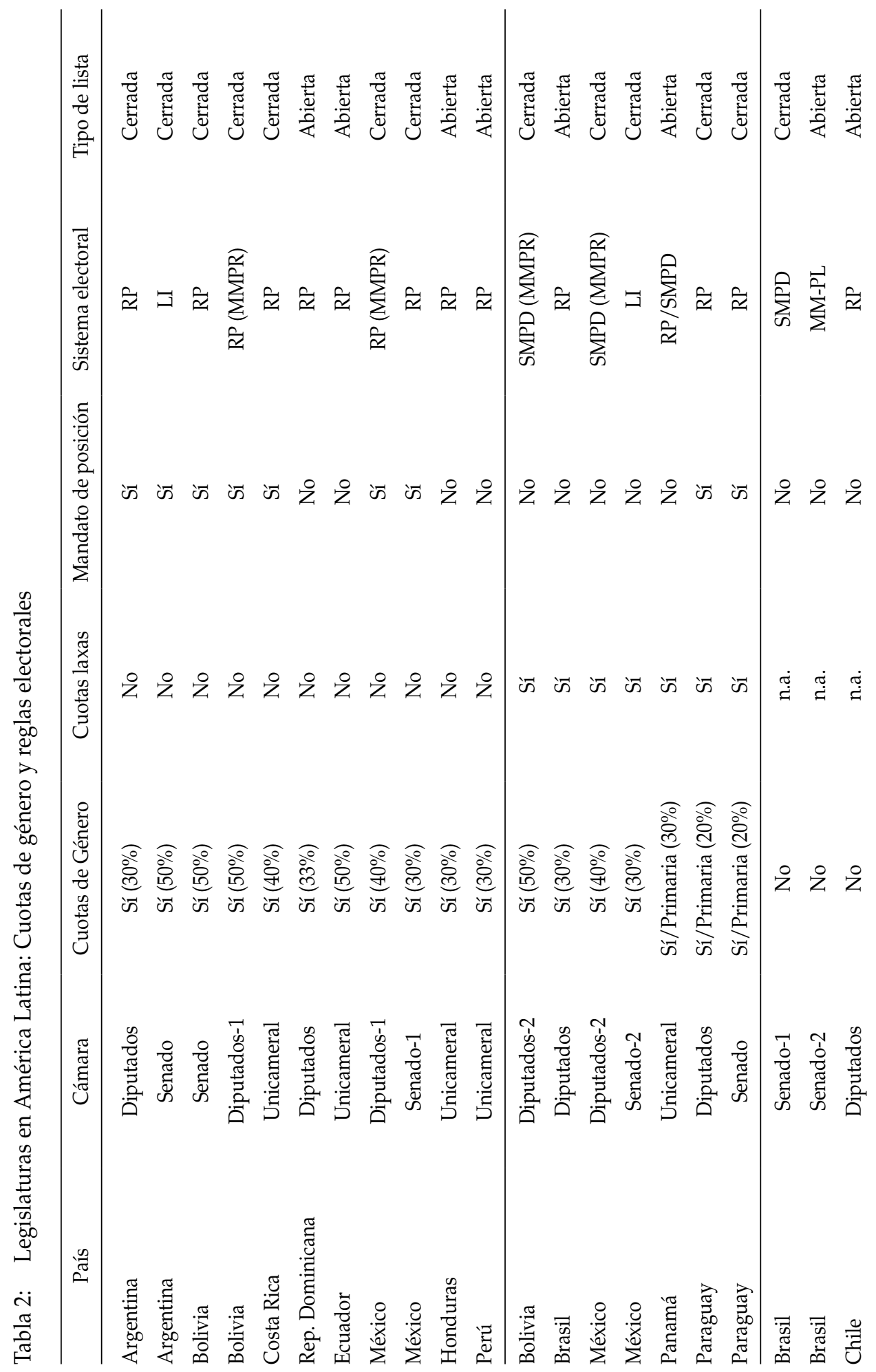




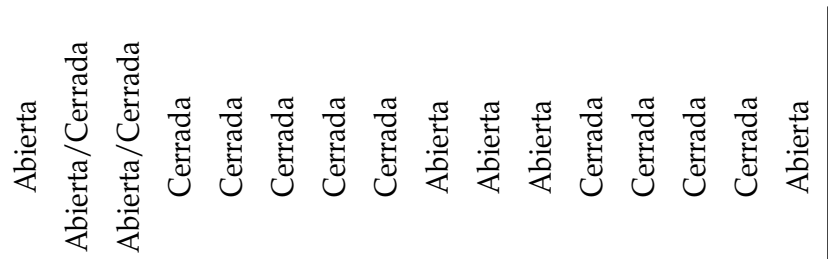

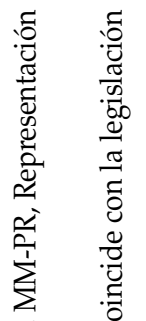

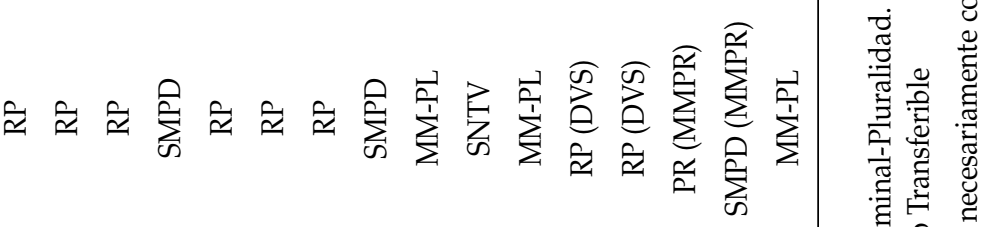

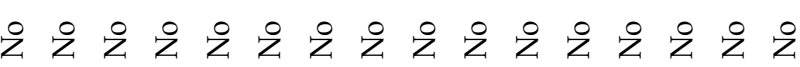

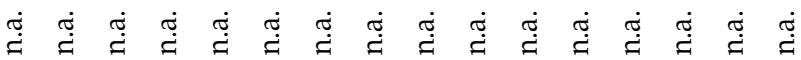

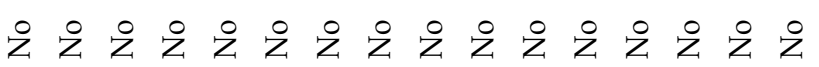

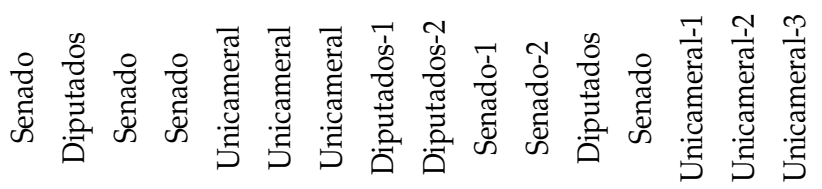

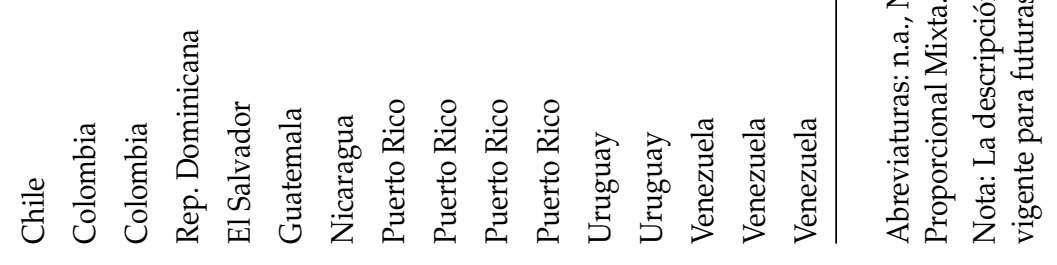


El primer grupo de regímenes de cuotas, que se compone de la Cámara de Diputados y el Senado argentinos, la Cámara de Diputados (escaños de RP solamente) y el Senado bolivianos, la Asamblea de Costa Rica, y las bancas proporcionales de la Cámara de Diputados y el Senado mexicanos, contiene cierta variación en su interior. Por un lado, en Argentina y Costa Rica los mandatos de posición se basan, en parte, en el pasado éxito electoral del partido político en el distrito: la práctica indica que uno de los dos primeros candidatos debe ser para una mujer, excepto en los distritos de gran magnitud donde el partido históricamente ha ganado tres o más escaños, en cuyo caso, una mujer puede ocupar la tercera posición en la lista y cumplir el mínimo exigido por la ley. Más aún, las posiciones subsiguientes de la lista deben ser asignadas con la misma lógica, de tal manera que el porcentaje de mujeres en puestos esperables en las listas de candidatos se corresponda con el porcentaje de la cuota. En cambio, en los casos de la Cámara de Diputados boliviana y las bancas proporcionales de la Cámara y el Senado mexicanos, las cuotas tienen un diseño menos complejo: se requiere que uno de cada dos miembros de la lista sea una mujer en las elecciones de diputados en Bolivia y México; y uno de cada tres en el Senado mexicano.

En el segundo grupo de regímenes de cuotas se incluyen las legislaturas unicamerales de Ecuador, Honduras y Perú, junto a la Cámara de Diputados de República Dominicana. En estos casos, las reglas exigen un porcentaje mínimo de mujeres, pero el uso de listas abiertas vuelve imposible poner en práctica mandatos de posición.

El último grupo se compone de casos donde existe legislación sobre cuotas, pero la legislación no exige un porcentaje mínimo de mujeres en las listas de candidatos o bien tienen otros defectos graves; e incluye: los escaños por pluralidad de la Cámara de Diputados de Bolivia, los diputados en Brasil, los diputados por pluralidad y los senadores por lista incompleta en México, la Asamblea en Panamá y ambas Cámaras en Paraguay. En Brasil, el porcentaje de la cuota se aplica al número total de posiciones de la lista que un partido puede presentar en un distrito, lo que va desde el $150 \%$ al $200 \%$ de los escaños a ser elegidos. Así, un partido teóricamente podría cumplir con la legislación mediante la presentación de una lista con sólo el 70\% del número de escaños en juego, aun cuando estuviera integrada sólo por hombres; por lo cual, en la práctica, el porcentaje de mujeres candidatas a la Cámara de Diputados, tal como era de esperar, ha sido muy bajo: en 2006, por ejemplo, la proporción de mujeres en las listas alcanzó apenas al 13\%. Para las bancas del Senado de México electas por lista incompleta, la legislación requiere que un mínimo del 30\% de los candidatos sean mujeres, pero autoriza a los partidos a colocarlas en cualquier distrito y en cualquier posición de la lista; así, por ejemplo, los líderes partidarios pueden cumplir con la ley haciendo competir a las mujeres en los distritos donde no esperan ganar o ubicarlas en el segundo lugar en aquellos otros donde esperan lograr una sola banca. Panamá exige a los partidos la realización de primarias intra-partidarias, en las que deben incluir un mínimo de mujeres (30\%) entre los candidatos, pero permite a los partidos no cumplir el requisito, si un número insuficiente de mujeres se inscriben como candidatas. Esta última laguna, así como la muy baja incidencia de las primarias en Panamá, ha hecho que esta ley sea en gran medida irrelevante (Jones, 2010). 
Por último, para la elección tanto de la Cámara de Diputados como del Senado en Paraguay, los partidos deben incluir, como mínimo, un 20\% de mujeres en las listas para las elecciones internas que son normalmente muy competitivas (sobre todo en el Partido Colorado, el más votado). Sin embargo, como Paraguay cuenta con distritos relativamente pequeños para la elección de la Cámara de Diputados y se utiliza representación proporcional para asignar los escaños en las elecciones primarias, la legislación de cuotas en la práctica no tiene ningún efecto real en la composición de la Cámara. La elección del Senado, por el contrario, se realiza en distrito nacional de 45 miembros lo cual podría facilitar la eficacia de las cuotas. Sin embargo, tal como fue el caso en la renovación de 2003, la combinación de un alto nivel de fragmentación tanto en las primarias de 2007 como en las elecciones generales de 2008 resultó en una incidencia moderada de la ley en la composición de las listas en las elecciones generales.

En términos generales, las leyes de cuotas han sido cumplidas en América Latina. No obstante, a los ocasionales incumplimientos, en otras oportunidades se ha sumado que la interpretación de la ley de cuotas ha estado en disputa entre organizaciones que promueven una visión amplia en la implementación de legislación y otros actores que sostienen una interpretación más restrictiva. La discusión anterior sobre el tercer grupo de sistemas de cuotas, sin embargo, pone de relieve varias lagunas y zonas grises presentes en las leyes de cuotas en algunos países, lo que llevó a que sean clasificadas como mal diseñadas o laxas (en la terminología utilizada en este artículo). Teniendo en cuenta la variedad de regímenes descrita anteriormente, las elecciones más recientes en estos 19 países ofrecen un laboratorio ideal para estudiar la eficacia relativa de las diferentes combinaciones de leyes de cuota de género y reglas electorales. En este estudio, el análisis de la población se compone de todas las listas (sean de partidos o alianzas electorales) a nivel de distrito que ganaron uno o más escaños. En total, las elecciones de estos 19 países ofrecen 1.469 listas (observaciones) que obtuvieron una o más bancas en un distrito con magnitud mayor a uno.

\section{VARIABLES DEPENDIENTE E INDEPENDIENTES}

En este trabajo la variable dependiente es el porcentaje de mujeres elegidas por partido/ alianza en determinado distrito; es decir, el número de mujeres legisladoras elegidas por partido en el distrito, sobre el número total de legisladores electos por el partido en el mismo distrito. Las variables independientes se dividen en cuatro grupos. El primer grupo está conformado por una serie de variables dicotómicas que dan cuenta de las cuatro combinaciones posibles de sistemas electorales y leyes de cuotas: (a) lista cerrada y ley de cuota de género bien diseñada con mandatos de posición (lista cerrada + cuota); (b) lista abierta y ley de cuota de género bien diseñada (lista abierta + cuota); (c) lista cerrada y ley de cuota de género laxa o sin ley de cuota de género (lista cerrada + cuota laxa/sin cuota) y; (d) lista abierta y ley de cuota de género laxa o sin ley de cuotas de género (lista abierta + cuota laxa/sin cuota). El análisis preliminar indica que los sistemas con cuotas laxas o mal diseñadas no difieren significativamente en su impacto sobre la elección de 
las mujeres de sistemas que carecen de cualquier tipo de leyes de cuotas, lo cual es un resultado esperable debido a los problemas de diseño que presentan tales leyes. ${ }^{9}$

El segundo grupo de variables da cuenta del tamaño de la magnitud partidaria; es decir, el número de escaños que el partido gana en un distrito electoral (Matland, 2005). Dada la falta de una teoría sólida que especifique la forma funcional de la relación entre la magnitud partidaria y la elección de las mujeres, la magnitud partidaria fue agrupada en una serie de categorías: 1, 2, 3, 4, 5, 6 a 10, 11 a 15, y de 16 a 28 (Beck et al., 1998). ${ }^{10}$ En América Latina, tal como muestra la Tabla 3, casi un tercio (31\%) de los legisladores en distritos plurinominales son los únicos representantes electos de su partido en el distrito; a la vez, un quinto (21\%) de los legisladores son uno de los dos únicos representantes de su partido en el distrito.

Tabla 3: Distribución de bancas obtenidas en América Latina $(\mathrm{M}>1)$

\begin{tabular}{ccc}
\hline $\begin{array}{c}\text { Número de bancas } \\
\text { obtenidas }\end{array}$ & $\begin{array}{c}\text { Distribución de las } \\
\text { observaciones }\end{array}$ & $\begin{array}{c}\text { Proporción del total } \\
\text { de bancas }\end{array}$ \\
\hline 1 & 888 & 31 \\
2 & 302 & 21 \\
3 & 119 & 12 \\
4 & 54 & 8 \\
5 & 27 & 5 \\
6 a 10 & 57 & 14 \\
11 a 15 & 15 & 5 \\
16 a 28 & 7 & 5 \\
\hline
\end{tabular}

Nota: Los 547 casos donde la magnitud de distrito es igual a 1 no se incluyen en esta tabla.

El tamaño de la magnitud partidaria de un determinado partido político (o alianza electoral) en un distrito electoral es resultado de múltiples factores. Si bien el determinante más importante es el tamaño de la magnitud del distrito, cuya correlación con la magnitud partidaria es de 0,52 en nuestra población, otros determinantes institucionales destacados incluyen: la fórmula de asignación de bancas legislativas, la presencia o ausencia de umbrales electorales a nivel nacional o a nivel de distrito, y en los sistemas presidenciales, el calendario de las elecciones presidenciales y legislativas y la fórmula electoral presidencial (Payne et al., 2007).

9 Si los sistemas de cuotas laxas son incluidos en la categoría "cuotas", los resultados son comparables a los aquí presentados, aunque la diferencia de rendimiento entre los sistemas de cuotas con lista cerrada y aquellos con listas abiertas se ensancha, al tiempo que la brecha de rendimiento se estrecha entre los sistemas con cuotas y sin cuotas.

10 La población incluye pocos casos de magnitud partidaria igual o mayor a 6, por lo tanto se utilizan tres categorías agrupadas: de 6 a 10, de 11 a 15, y de 16 a 28. 
Tercero, nuestro análisis controla los efectos del partido político en la elección de las mujeres en estos países. La literatura ha señalado que los partidos ubicados a la izquierda en el espectro político contribuyen desproporcionadamente al contingente femenino en las legislaturas de Europa (Caul Kittilson, 2006). Para este estudio, la posición programática de los partidos fue operacionalizada como una variable dicotómica, donde los partidos de izquierda fueron codificados como 1; y los restantes, 0 . Los datos provienen de la posición ideológica de los partidos latinoamericanos elaborada en Alcántara (2008), junto a actualizaciones posteriores publicadas por el Observatorio de Elites Parlamentarias de América Latina a partir de nuevos relevamientos. Los partidos políticos no cubiertos por Alcántara (2008) ni por las siguientes actualizaciones han sido codificados a partir de fuentes secundarias siguiendo el mismo marco general utilizado por Alcántara. ${ }^{11}$

Por último, la elección de la mujer puede verse influenciada por una serie de factores contextuales, tales como la participación relativa de mujeres en el mercado laboral, el nivel de igualdad entre géneros en los logros educativos, el nivel de desarrollo económico, y las actitudes hacia la capacidad de las mujeres políticas (Inglehart y Norris, 2003; Matland, 1998; Studlar y McAllister, 2002). En ese sentido, un conjunto de cinco variables se emplea para controlar el impacto potencial de estos factores contextuales. Primero, la participación en el "Mercado Laboral" es la relación entre la proporción de mujeres y hombres en la fuerza de trabajo (PNUD, 2011). Segunda, la variable "Educación Secundaria" ha sido definida como la relación entre la proporción de mujeres y la proporción de hombres que poseen (al menos) título secundario como máximo nivel educativo alcanzado (PNUD, 2011). Tercero, la variable "Educación Superior" es la relación entre la proporción de mujeres con estudios superiores o universitarios (completos e incompletos) respecto a la proporción de hombres (Latinobarómetro, 2009). Cuarto, el nivel de "Desarrollo Económico" ha sido definido como el PIB per cápita, expresado en miles de dólares estadounidenses, por su paridad de poder de compra (PNUD, 2011). Quinto, la "Opinión Pública" se mide como el grado de acuerdo con la frase "Los hombres son mejores líderes políticos que las mujeres". La pregunta, tomada de la Encuesta Mundial de Valores (WVS, por su sigla en inglés), fue medida por Latinobarórmetro en 2009 para toda América Latina. ${ }^{12}$

\section{ANÁLISIS Y RESULTADOS}

El análisis en esta sección utiliza regresión lineal por mínimos cuadrados ordinarios (OLS) con errores estándares agrupados, cuyos resultados no diferirían significativamente si la variable dependiente fuera transformada en forma logarítmica. Más aún, la decisión de emplear esta metodología se tomó luego de considerar una amplia gama de otras

11 Además de la posición ideológica, el análisis introdujo una segunda variable partidaria a fin de controlar la presencia de cuotas de género en las normas internas del partido (véase, Quota Project, 2012). Sin embargo, esta variable está altamente correlacionada con la ideología partidaria: las cuotas son adoptadas mayormente por partidos de izquierda. Por ese motivo, esta variable tiene menor poder explicativo y no se incluye en este análisis.

12 Los encuestados deben expresar su nivel de acuerdo: (1) muy de acuerdo, (2) de acuerdo, (3) en desacuerdo y (4) muy en desacuerdo. Para Puerto Rico, los datos provienen de WVS 1999-2004. 
estrategias de análisis empírico. Primero, el número limitado de países, junto con la cantidad relativamente pequeña de observaciones dentro de cada uno de ellos torna este conjunto de datos inadecuados para un enfoque multinivel. Segundo, métodos alternativos como distribuciones Poisson o Negative-binomial (utilizando el número de mujeres elegidas como la variable dependiente) resultan subóptimos debido a que no se ajustan a la distribución de los datos. Dada la cantidad de observaciones, el uso de una regresión lineal no sufre problemas equivalentes asociados a las asunciones de la distribución. Tercero, los resultados usando tanto regresiones ordered-probit como orderedlogit son comparables a los aquí presentados. Sin embargo, el número limitado de casos en los que las mujeres ganan dos o más escaños en un distrito genera problemas con la estimación de los puntos de corte de mayor valor. Por ese motivo, ambos tipos de regresión no fueron considerados métodos viables para la estimación.

\section{ANÁLISIS SOBRE EL TOTAL DE LA POBLACIÓN}

La Tabla 4 presenta los resultados del análisis de la población total. Tal como puede observarse, ninguna de las cinco variables contextuales presenta algún impacto significativo en el porcentaje de mujeres electas, tampoco afecta los coeficientes de las otras variables. Por lo tanto, la discusión del análisis se centra en el Modelo 6. Las tres variables del sistema electoral son significativas al nivel de 0,001 . Los coeficientes estimados son negativos, lo que indica que los tres sistemas electorales resultan, ceteris paribus, en un porcentaje significativamente menor de mujeres electas comparado con un sistema con cuotas de género y listas cerradas, que es el baseline de la regresión.

Para una mejor comparación de los cuatro sistemas electorales, se presentan los valores esperados (e intervalos de confianza al 95\%) para las cuatro variables, incluyendo las variables ajenas al sistema electoral en sus valores promedio. El porcentaje esperado más alto de mujeres electas por un partido en un distrito electoral se encuentra entre los sistemas de lista cerrada con cuotas, con un valor esperado de 33\% (y un intervalo de confianza del $95 \%$ entre $31 \%$ y $36 \%$ ), seguido por los sistemas de lista abierta con cuotas con un valor esperado de 19\% (e intervalo de confianza de 15\% a 22\%). Por detrás aparecen los sistemas con cuotas mal diseñadas (laxas) o sin cuotas usando listas cerradas, con un valor esperado de $14 \%$ (12\% a $16 \%$ ); y luego, aquellos casos con cuotas mal diseñadas (laxas) o sin cuotas usando listas abiertas, con un valor esperado de 13\% (10\% a 17\%).

Observando una población total similar, Jones (2009) extrae tres conclusiones respecto a la relación entre el tipo de lista, leyes de cuota y la elección de mujeres. En primer lugar, un porcentaje significativamente mayor de mujeres fueron elegidas ( $8 \%$ más en promedio) en sistemas de cuotas con listas cerradas que en otros donde las cuotas fueron implementadas con listas abiertas. En segundo lugar, la presencia de leyes de cuotas bien diseñadas, independientemente del tipo de lista, resultó en un mayor porcentaje de mujeres electas que en los sistemas con una normativa laxa o ninguna ley de cuotas. La diferencia más grande se encontró entre los sistemas con cuotas y listas cerradas, que en promedio resultaron en un $20 \%$ más de mujeres electas que en casos con cuotas 
laxas o sin cuotas y listas cerradas; y en un 17\% más respecto a otros con cuotas laxas o sin cuotas y listas abiertas. Y los sistemas de cuotas con listas abiertas resultaron en un porcentaje esperado de mujeres electas 13\% y 10\% mayor, respectivamente. En tercer lugar, no se observó ninguna diferencia significativa en el porcentaje de mujeres que se esperaba fueran electas con listas cerradas y abiertas con leyes de cuotas mal diseñadas o sin leyes de cuota.

El análisis realizado aquí ofrece conclusiones similares a los puntos primero y tercero, pertenecientes al análisis de Jones (2009), presentados en el párrafo anterior. En primer lugar, el uso de cuotas combinado con listas cerradas resulta en una mayor proporción de mujeres electas. Este resultado es notablemente superior a cualquiera de las otras combinaciones de reglas electorales y leyes de cuotas. En segundo lugar, los tipos de listas no presentan diferencias en su impacto cuando no hay cuotas en vigencia ni cuando se combinan con leyes de cuotas laxas. No obstante, el presente análisis sugiere una conclusión distinta respecto a la superioridad de las cuotas con listas abiertas vis$\grave{a}$-vis los casos con cuotas laxas o sin cuotas. Mientras que Jones (2009) encontró que la proporción de mujeres electas es significativamente mayor en sistemas de cuotas con lista abierta, el presente análisis no pudo detectar diferencias significativas. Es decir, mientras que el uso de las cuotas bien diseñadas en conjunto con las listas abiertas dio lugar a una proporción de mujeres electas mayor que la encontrada en los sistemas con cuotas laxas o sin cuotas (ya sea en listas abiertas y cerradas), el solapamiento de los intervalos de confianza en el presente análisis indica que la diferencia entre estos sistemas no es tan importante ni significativa como la que se encontró anteriormente.

Por último, al igual que Jones (2009), la evidencia indica que la ideología del partido político se relaciona notablemente con el porcentaje de mujeres electas; el cual es significativamente mayor de mujeres legisladoras que son electas entre los partidos clasificados de izquierda comparado con los otros partidos políticos. El valor esperado de mujeres electas para los partidos de izquierda es $24 \%$, comparado con el $15 \%$ para todos los demás partidos.

\section{ANÁLISIS DE LA SUBPOBLACIÓN}

Para una mejor comprensión de la relación existente entre las reglas electorales, ley de cuotas y la elección de mujeres, esta sección amplía el análisis anterior en dos direcciones. En primer lugar, la población bajo estudio se divide en cinco subgrupos definidos sobre la base del valor de magnitud partidaria. En segundo lugar, se divide la misma población en cuatro subgrupos según la combinación de reglas electorales y leyes de cuotas. Cada subgrupo es, luego, examinado en forma independiente.

La Tabla 5 presenta el análisis para los cinco subgrupos según magnitud partidaria. ${ }^{13}$ Dado que la magnitud partidaria se mantiene constante en los Modelos 7 a 10, esta

13 Los casos con magnitud partidaria con valores entre 5 y 28 se examinan de manera conjunta debido al pequeño número de casos en este rango. 


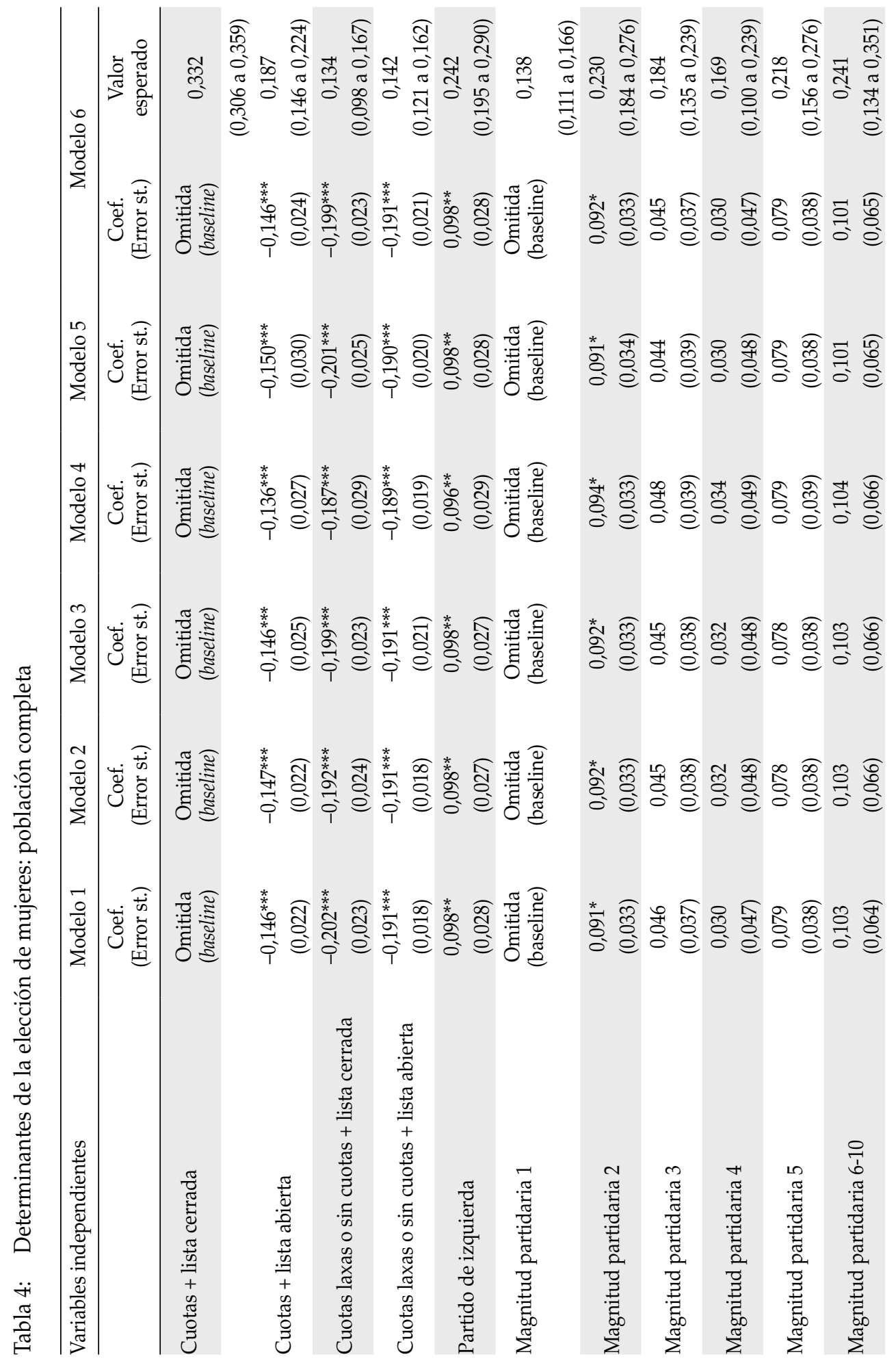




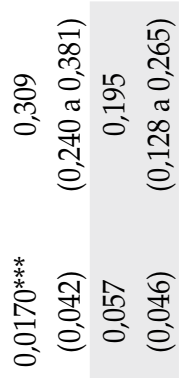

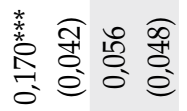

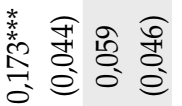

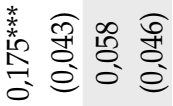

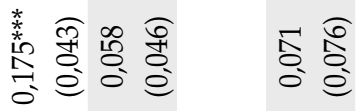

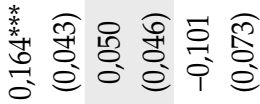

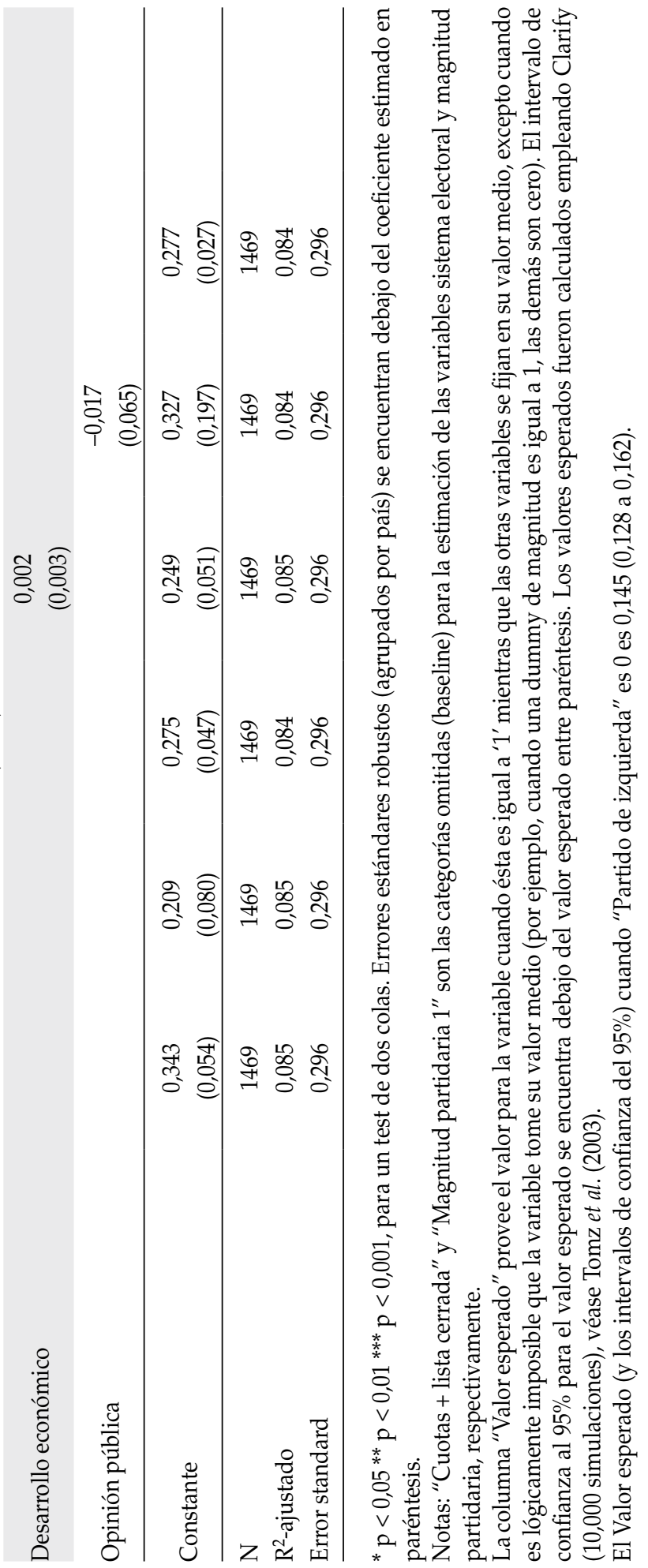


variable sólo es incorporada en el Modelo 11. No obstante, por razones de espacio y por la falta de diferencias significativas, los resultados no aparecen reportados en la Tabla.

Los resultados de la Tabla 5 ofrecen una interpretación más matizada de la relación entre el sistema electoral, leyes de cuotas y la elección de mujeres que la presentada en el análisis previo de la población total. En primer lugar, la superioridad de los sistemas con cuota comparada con los sistemas con cuotas laxas o sin cuotas varía en función de la magnitud partidaria. Cuando la magnitud partidaria es mínima, las listas cerradas y abiertas son equivalentes en cuanto a la elección de las mujeres, al tiempo que los sistemas con cuotas laxas y sin cuotas son también equivalentes con independencia del tipo de lista (Modelo 7). Este patrón es similar al hallado en el estudio anterior.

Mientras la evidencia presentada por Jones (2009) indicaba la amplia superioridad de los sistemas de cuotas respecto a los casos con cuotas mal diseñadas o sin cuotas en cuanto a la elección de mujeres, con indiferencia del tipo de lista, ${ }^{14}$ la evidencia analizada en el presente trabajo sugiere un patrón similar sólo para los casos donde las cuotas son implementadas con listas cerradas. Estos casos en los modelos 8-10 siempre resultan en la elección de una proporción significativamente mayor de mujeres que en los sistemas con cuotas mal diseñadas o sin cuotas. No obstante, en contraste con los resultados anteriores, la evidencia aquí presentada indica que el uso de cuotas con listas abiertas en varios modelos no se diferencia sustantivamente de los sistemas con cuotas laxas o sin cuotas, en especial en los modelos 8 y 10.

En ese sentido, los resultados de Jones (2009) indicaban que la mayor eficacia de las cuotas con listas cerradas respecto a su implementación con las listas abiertas se limitaba a los casos donde la magnitud partidaria era de dos y, en menor medida, tres escaños. En cambio, para otras magnitudes partidarias (es decir, uno y cuatro o más escaños), no existían diferencias significativas en el porcentaje esperado de mujeres electas en sistemas de cuotas, cualquiera fuera el tipo de lista. La evidencia aquí presentada ofrece una conclusión similar: mientras se observa una brecha importante en el rendimiento de las cuotas (de acuerdo al tipo de lista) cuando la magnitud partidaria es de dos escaños, la diferencia relativa es modesta para los otros casos; la única excepción ocurre cuando la magnitud partidaria es cuatro, pero el número de casos es pequeño y los intervalos de confianza se vuelven más grandes.

En suma, las diferencias observadas en el porcentaje esperado de mujeres electas cuando las cuotas se implementan con diferentes tipos de lista es, principalmente, el resultado del rendimiento de las cuotas cuando la magnitud partidaria es de dos escaños: el valor esperado para las listas cerradas es de $50 \%$, lo que implica 29 puntos más que el valor esperado para las listas abiertas. La fuente principal de esta diferencia es que la implementación de cuotas con lista cerrada, cuando la magnitud partidaria es dos, en casi todos los casos asegura un piso mínimo efectivo de $50 \%$, dado que la ley requiere que al menos uno de los dos candidatos sea una mujer. Sin embargo, la implementación con listas abiertas no

14 Los hallazgos presentaban como excepción los casos donde la magnitud partidaria era cuatro; y aquellos otros donde el pequeño número de observaciones dio lugar a valores esperados imprecisos. 


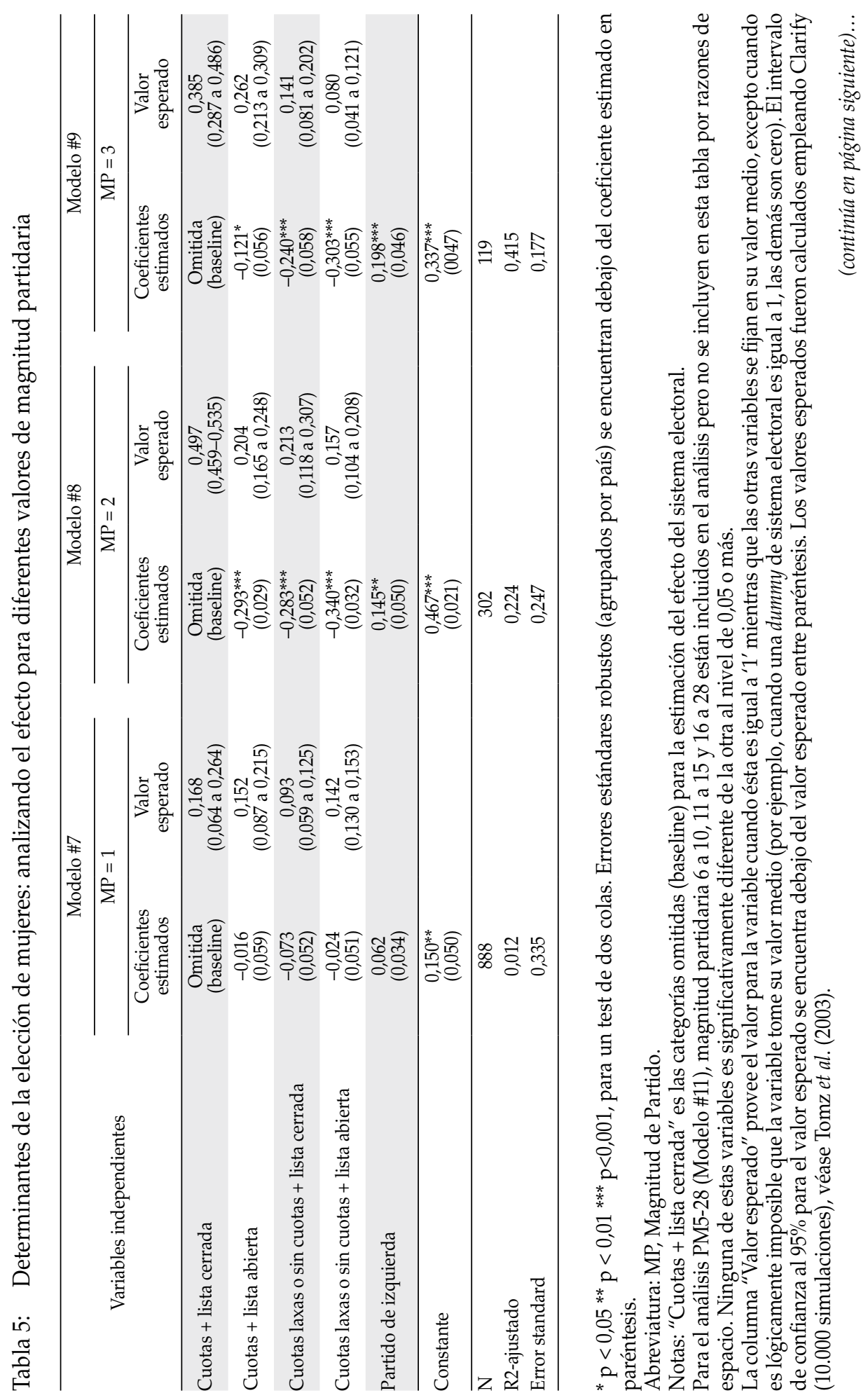




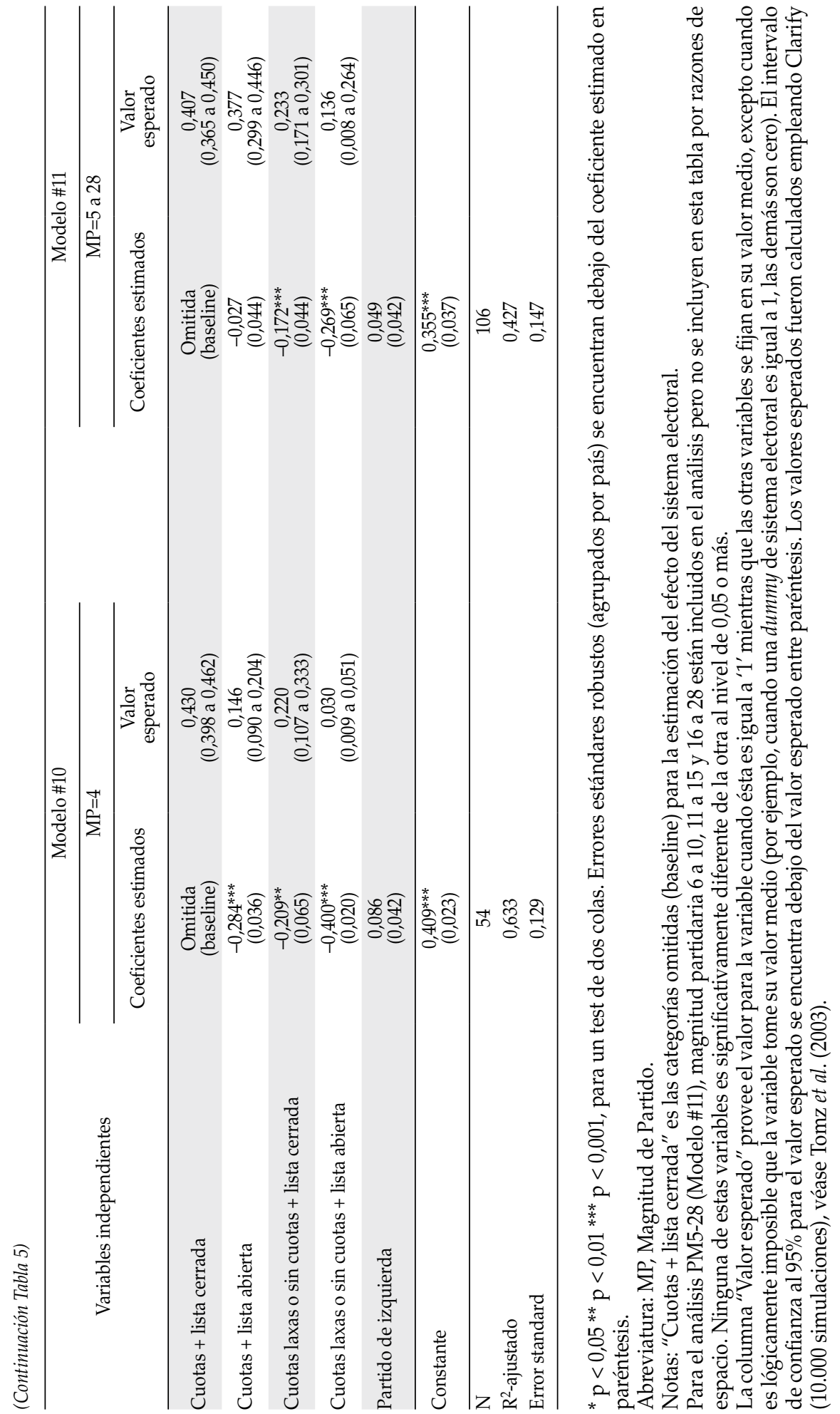


tiene ningún piso mínimo y el porcentaje potencial de mujeres electas comprende todos los valores hasta $0 \%$.

La Tabla 6 presenta el análisis según subgrupos en función del tipo de lista y legislación de cuotas vigente. Es decir, los cuatro tipos de reglas electorales y tipos de cuotas examinados anteriormente: (a) lista cerrada + cuota (Modelo 12), (b) lista abierta + cuota (Modelo 13), (c) lista cerrada + cuota laxa/sin cuota (Modelo 14), y (d) lista abierta + cuota laxa/sin cuota (Modelo 15). Este análisis proporciona información crucial sobre la medida en que el efecto de la magnitud partidaria en la elección de las mujeres varía dependiendo del tipo regla electoral. ${ }^{15}$ Debido al número limitado de casos con una magnitud superior a cinco, las tres categorías superiores de magnitud partidaria de la Tabla 4 se fusionan en un solo grupo (magnitud partidaria de 6 a 28 escaños). ${ }^{16}$

La evidencia aquí presentada sugiere una conclusión similar a la ofrecida en el análisis anterior (cfr. Jones, 2009) respecto a la relación entre magnitud partidaria y elección de mujeres. En aquel trabajo no se encontró ninguna relación significativa entre estas variables, excepto en la implementación de cuotas con listas cerradas donde se descubrió una relación en alguna medida curvilínea (con magnitud partidaria de dos escaños representando el pico de la curva). De manera similar, en este análisis se observa una relación relativamente vaga entre la magnitud partidaria y la elección de las mujeres en los cuatro sistemas.

Las principales excepciones a la conclusión anterior giran en torno al limitado éxito de las mujeres en los casos en que los partidos logran un solo diputado en el distrito en elecciones con listas cerradas, tanto en casos con cuotas como en otros con cuotas laxas o sin cuotas. La diferencia es particularmente notable cuando las cuotas se implementan con listas cerradas: mientras el valor esperado de la proporción de mujeres electas es apenas del $15 \%$ cuando la magnitud partidaria es uno, ese valor escala a 50\% cuando la magnitud partidaria es dos; y a partir de magnitudes partidarias de tres y más, la proporción de mujeres declina levemente, pero el intervalo de confianza se mantiene en el rango de $47 \%$ a $53 \%$, rodeando la estimación de $50 \%$ para cuando la magnitud de los partidos es de dos escaños. ${ }^{17}$

A su vez, también existe una notable, aunque no siempre significativa, tendencia positiva en la relación entre la magnitud partidaria y la elección de la mujer en los sistemas con cuotas y lista abierta. Así, con la excepción de los casos con magnitud partidaria de cuatro escaños, el porcentaje de mujeres elegidas aumenta progresivamente a medida que aumenta la magnitud partidaria, con un porcentaje de mujeres electas significativamente

15 También es posible examinar el efecto diferencial de la magnitud partidaria en un análisis de la población total utilizando términos de interacción. Sin embargo, la interpretación de los efectos diferenciales de las combinaciones de magnitud partidaria-cuota/sistema electoral se ve gravemente socavada por el número muy grande de términos de interacción necesarios para este análisis. La misma línea de razonamiento es válida en relación con el análisis presentado en la Tabla 4.

16 El número relativamente pequeño de casos con valores de magnitud partidaria de cuatro o más requiere que tratemos a los resultados de la Tabla 5 para estas categorías con cierta cautela.

17 El número de observaciones donde la magnitud del partido es cuatro es pequeño en nuestra población, lo cual limita la capacidad de hacer inferencias seguras a partir de los resultados obtenidos. 


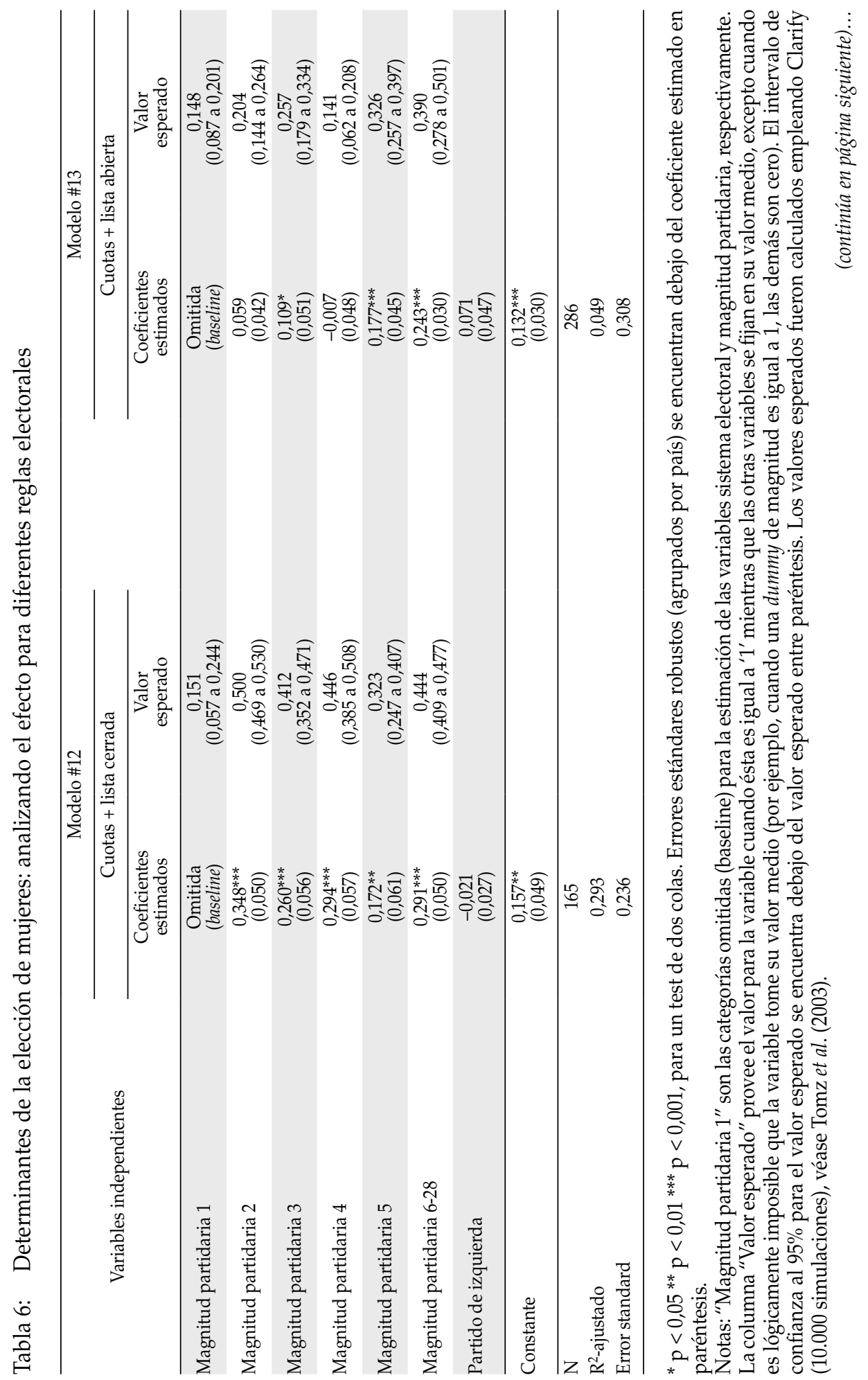




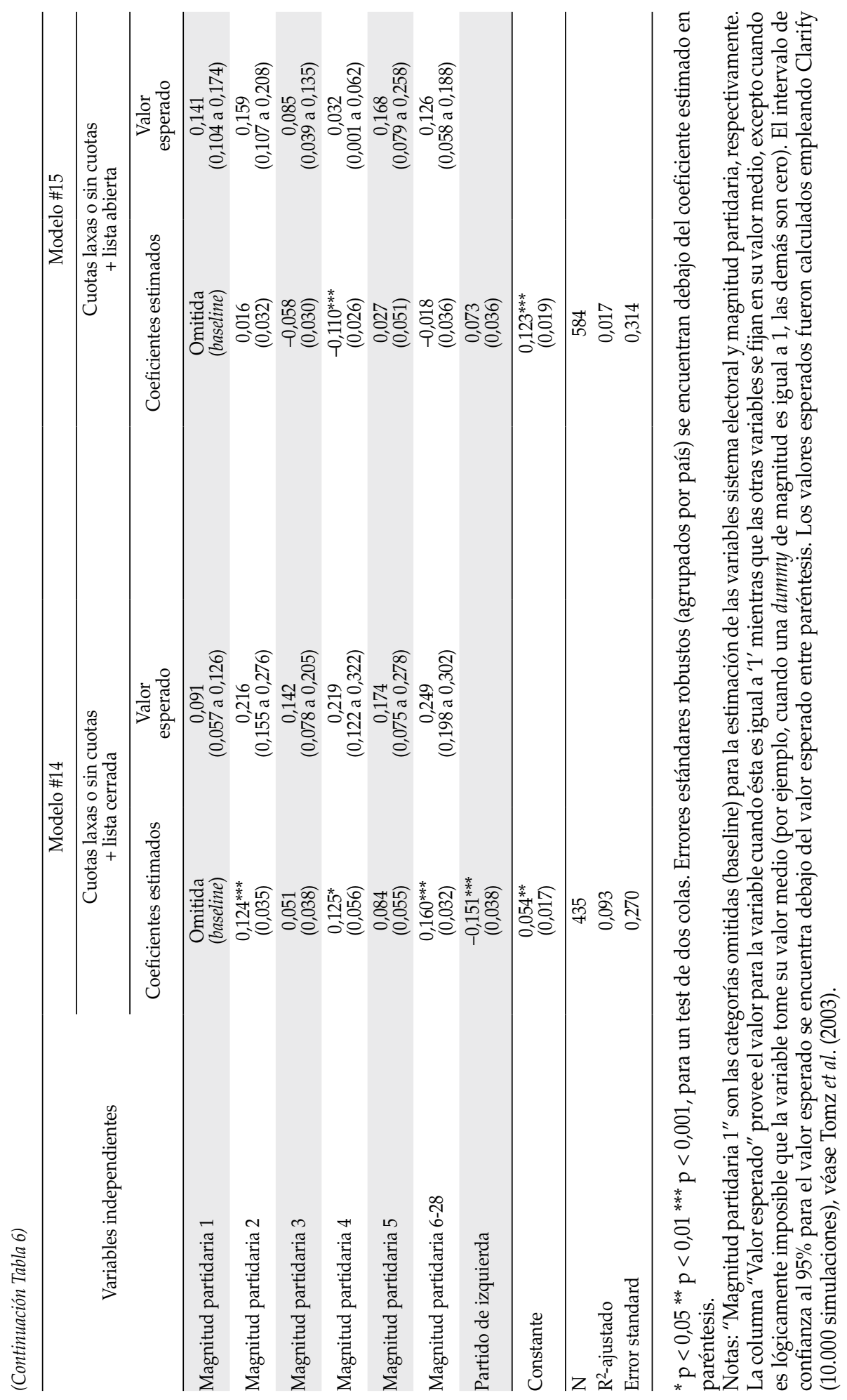


mayor cuando la magnitud partidaria es de cinco o más, en comparación a cuando la magnitud es uno.

Por último, como muestra la Tabla 6, la ideología partidaria tiene un impacto positivo y significativo en la elección de las mujeres en los sistemas con cuotas laxas o sin cuotas (modelos 14 y 15), pero no en los dos sistemas con cuotas (Modelos 12 y 13). Estos resultados sugieren que, en ausencia de leyes de cuotas bien diseñadas, la ideología del partido juega un papel mucho más relevante en cuanto a la elección de las mujeres que en los casos en los que cuotas bien diseñadas están en vigencia. En otras palabras, la existencia de leyes de cuotas que se aplican sistemáticamente a todos los partidos reduce cualquier diferencia basada en la ideología en cuanto a su apertura a elegir mujeres para cargos legislativos.

\section{CONCLUSIÓN}

Las leyes de cuotas de género son una herramienta indispensable para los países que desean incrementar drásticamente la presencia de mujeres en sus legislaturas nacionales, provinciales y locales en el corto y mediano plazo. En línea con el análisis desarrollado en Jones (2009), el presente trabajo, utilizando datos de los últimos comicios legislativos en América Latina, pone de relieve que la probabilidad de que mujeres candidatas sean electas es mucho mayor en sistemas de representación proporcional, MMPR y semiproporcional donde la legislación de cuotas está bien diseñada, especialmente cuando se combinan con listas cerradas.

Independientemente del tipo de lista (cerrada o abierta), la presencia de leyes de cuotas bien diseñadas resulta en promedio en un porcentaje significativamente mayor de mujeres legisladoras electas comparado con los casos en los que esta normativa está ausente (aun empleando el mismo tipo de lista). La lección que se desprende de estos resultados en términos de política pública es clara para los defensores de una mayor representación legislativa de las mujeres en países que carecen de una legislación de cuotas bien concebida y emplean sistemas electorales de representación proporcional, MMPR o semiproporcional: la adopción de una legislación de cuotas bien diseñada importa.

La literatura convencional sostiene que las cuotas de género, cuando están bien diseñadas funcionan mejor con listas cerradas que con listas abiertas. La evidencia aquí presentada indica que esta idea es correcta: en promedio, un porcentaje mayor de mujeres tienen chances de ser electas cuando, ceteris paribus, se utilizan listas cerradas en lugar de listas abiertas. No obstante, aunque las listas cerradas son superiores a las listas abiertas en esta materia, la diferencia entre ambos arreglos institucionales no es tan abrumadora como a menudo se supone (aunque en este estudio resulta un poco mayor que la encontrada en el análisis de 2009); y es principalmente la consecuencia de la marcada superioridad de los sistemas de listas cerradas cuando un partido elige a dos legisladores en un distrito.

La relación entre la magnitud del partido y el porcentaje de mujeres electas varía considerablemente dependiendo del tipo de lista utilizada (abierta o cerrada) y la 
presencia o ausencia de leyes de cuotas bien diseñadas. En los sistemas de listas abiertas (especialmente con cuotas laxas o sin cuotas) la magnitud partidaria no se relaciona de manera significativa con el porcentaje de mujeres elegidas. La relación más destacada entre la magnitud partidaria y la elección de mujeres se encuentra en los sistemas que combinan leyes de cuotas bien diseñadas y listas cerradas. En estos sistemas, es particularmente importante si la magnitud partidaria es uno, porque en estos casos, la probabilidad de que una candidata resulte electa es comparativamente muy baja. Esto pone de relieve no sólo la tendencia de los partidos a situar a sus figuras masculinas como cabeza de lista, sino también el cumplimiento mínimo que intentan dar a las leyes de cuotas, colocando a las candidatas en la segunda o tercera posición, según les permita la legislación vigente.

Pocas legislaturas de América Latina, tales como las dos cámaras del Congreso argentino, el Senado boliviano o la Asamblea Legislativa costarricense, eligen sus miembros empleando combinaciones de leyes de cuotas y sistemas electorales de manera casi ideal para impulsar el ingreso de mujeres. Así, como resultado de esta combinación de reglas, estas legislaturas cuentan con un porcentaje de mujeres legisladoras que van desde el $37 \%$ al $47 \%$ en la actualidad; mientras que, lamentablemente, en más de la mitad de las 29 cámaras legislativas examinadas aquí, las mujeres representan actualmente menos del 20\% de los legisladores, y en más de un tercio de las legislaturas de las mujeres representan menos del 15\% de los miembros. Para esta gran mayoría de los países, las principales lecciones de este artículo proporcionan un modelo para futuras reformas destinadas a aumentar la presencia de mujeres en el Parlamento e identifican las potenciales limitaciones de las reformas en función de la interacción de las leyes de cuotas, el formato de lista del partido, y los valores de la magnitud partidaria.

\section{REFERENCIAS}

Alcántara Sáez, Manuel, ed. 2008. Politicians and Politics in Latin America. Boulder, CO: Lynne Rienner Publishers.

Bacchi, Carol. 2006. "Arguing For and Against Quotas: Theoretical Issues". Women, Quotas, and Politics, editado por Drude Dahlerup. New York: Routledge, 32-51.

Baldez, Lisa. 2007. "Primaries vs. Quotas: Gender and Candidate Nominations in Mexico, 2003". Latin American Politics and Society, 49 (3): 69-96.

Beck, Nathaniel, Jonathan N. Katz y Richard Tucker. 1998. “Taking Time Seriously: Time-Series-Cross-Section Analysis with a Binary Dependent Variable". American Journal of Political Science, 42 (4): 1260-1288.

Caul Kittilson, Miki. 2006. Challenging Parties, Changing Parliaments: Women and Contemporary Elected Office in Western Europe. Columbus: Ohio State University Press.

Dalherup, Drude. 2006. "Introduction". Women, Quotas, and Politics, editado por Drude Dahlerup. New York: Routledge, 3-31.

Dahlerup, Drude. 1998. "El uso de cuotas para incrementar la representación política de la mujer". Mujeres en el Parlamento. Más allá de los números, editado por M. Méndez Montalvo y J. Ballington. International IDEA, 2002.

Dahlerup, Drude y Lenita Freidenvall. 2005. "Quotas as «Fast Track» to Equal Representation for Women". International Feminist Journal of Politics, 7 (1): 26-48.

Franceschet, Susan, Mona Lena Krook y Jennifer M. Piscopo, eds. 2012. The Impact of Gender Quotas. New York: Oxford University Press. 
Htun, Mala N. y Mark P. Jones. 2002. "Engendering the Right to Participate in Decision-making: Electoral Quotas and Women's Leadership in Latin America". Gender and the Politics of Rights and Democracy in Latin America, editado por Nikki Craske y Maxine Molyneux. New York: Palgrave, 32-56.

IDEA. 2003. "Estudios comparativos sobre las cuotas de género". La aplicación de las cuotas: experiencias latinoamericanas. Lima: International IDEA.

Inglehart, Ronald y Pippa Norris. 2003. Rising Tide: Gender Equality and Cultural Change. New York, NY: Cambridge University Press.

Jones, Mark P. 2010. “La Representación de las Mujeres en la Asamblea Nacional de Panamá”. Las Reformas Electorales en Panamá: Claves de Desarrollo Humano Para la Toma de Decisiones, editado por Harry Brown Araúz. Ciudad de Panamá: Programa de las Naciones Unidas para el Desarrollo, 275-316.

Jones, Mark P. 2009. “Gender Quotas, Electoral Laws, and the Election of Women: Evidence from the Latin American Vanguard". Comparative Political Studies, 42 (1): 56-81.

Jones, Mark P. 2004. "Quota Legislation and the Election of Women: Learning from the Costa Rican Experience". Journal of Politics, 66 (4): 1203-1223.

Krook, Mona Lena. 2009. Quotas for Women in Politics. New York: Oxford University Press.

Larserud, Stina y Rita Taphorn. 2007. Designing for Equality: Best-Fit, Medium-Fit, and Non-Favourable Combinations of Electoral Systems and Gender Quotas. Stockholm: International IDEA.

Marx, Jutta, Jutta Borner y Mariana Caminotti. 2007. Las Legisladoras: Cupos de Género y Política en Argentina y Brasil. Buenos Aires: Siglo XXI.

Matland, Richard E. 1998. “Women's Representation in National Legislatures: Developed and Developing Countries". Legislative Studies Quarterly, 23 (2): 109-25.

Matland, Richard E. 2006. "Electoral Quotas: Frequency and Effectiveness". Women, Quotas, and Politics, editado por Drude Dahlerup. New York: Routledge, 275-292.

Matland, Richard E. 2005. “Enhancing Women's Political Participation: Legislative Recruitment and Electoral Systems". Women in Parliament: Beyond Numbers, A Revised Edition, editado por Julie Ballington y Azza Karam. Stockholm: International IDEA, 93-111.

Norris, Pippa. 2004. Electoral Engineering: Voting Rules and Political Behavior. New York: Cambridge University Press.

Paxton, Pamela, Melanie M. Hughes y Matthew A. Painter II. 2010. “Growth in Women's Representation: A Longitudinal Exploration of Democracy, Electoral System and Gender Quotas". European Journal of Political Research, 49 (1): 25-52.

Payne, J. Mark, Daniel Zovatto G. y Mercedes Mateo Díaz. 2007. Democracies in Development: Politics and Reform in Latin America (Expanded and Updated Edition). Washington, D.C.: Inter-American Development Bank.

Ríos Tobar, Marcela, ed. 2008. Mujer y Política: El Impacto de las Cuotas de Género en América Latina. Santiago de Chile: International IDEA, FLACSO-Chile, y Editorial Catalonia.

Schmidt, Gregory D. 2009. "The Election of Women in PR List Systems: Testing the Conventional Wisdom". Electoral Studies, 28: 190-203.

Schwindt-Bayer, Leslie. 2010. Political Power and Women's Representation in Latin America. New York: Oxford University Press.

Schwindt-Bayer, Leslie A. 2007. "Women in Power: How Presence Affects Politics". Paper prepared for the conference "Women in the Americas: Paths to Political Power". Inter-American Dialogue, InterAmerican Development Bank, League of Women Voters of the United States, and Organization of American States. Washington, D.C., March 28.

Studlar, Donley y Ian McAllister. 2002. “Does a Critical Mass Exist? A Comparative Analysis of Women's Legislative Representation since 1950". European Journal of Political Research, 41 (2), 233-253.

Thames, Frank C. y Maggie S. Williams. 2010. “Incentives for Personal Votes and Women's Representation in Legislatures". Comparative Political Studies, 43 (12): 1575-1600.

Tomz, Michael, Jason Wittenberg y Gary King. 2003. Clarify: Software for Interpreting and Presenting Statistical Results. Boston, MA: Harvard University.

Tripp, Aili Mari y Alice Kang. 2008. "The Global Impact of Quotas: On the Fast Track to Increased Female Legislative Representation". Comparative Political Studies, 41 (3): 338-61. 
United Nations. 1995. Report on the Fourth World Conference on Women: Beijing, September 4-15. New York: United Nations.

United Nations Development Programme. 2011. Human Development Report 2011. Sustainability and Equity: A Better Future for All. New York: United Nations Development Programme.

\section{Bases de datos}

Latinobarómetro. 2009. Latinobarómetro 2009. Corporación Latinobarómetro: Santiago de Chile. Disponible en: <http://www.latinobarometro.org/>, revisado: 01/04/2012.

Quota Project. 2012. Global Database of Quotas for Women. Stockholm: International IDEA and Stockholm University. Disponible en: <http:/ / www.quotaproject.org>, revisado: 01/02/2012.

Universidad de Salamanca. Observatorio de Elites Parlamentarias de América Latina. Disponible en: <http:/ / americo.usal.es/oir/elites/>, revisado: 01/02/2012.

Mark P. Jones. Joseph D. Jamail Chair en Estudios Latinoamericanos, Investigador en Ciencia Política del James A. Baker III Instituto para Políticas Públicas, y Director del Departamento de Ciencia Política de Rice University (Houston, Texas, EE.UU.).

E-mail: mpjones@rice.edu

Santiago Alles. Licenciado en Ciencia Política de la Universidad Católica Argentina y Magíster en Estudios Latinoamericanos de la Universidad de Salamanca. Actualmente estudiante de Ph.D. en Rice University.

E-mail: Santiago.Alles@rice.edu

Carolina Tchintian. Licenciada en Ciencia Política de la Universidad de Buenos Aires y Candidata a Magíster en Políticas Públicas de la Universidad Torcuato Di Tella. Actualmente estudiante de Ph.D. en Rice University.

E-mail: Carolina.R.Tchintian@rice.edu 
